\title{
Investigation of the stereochemical course of ene reductase-catalysed reactions by deuterium labelling
}

\author{
Elisabetta Brenna $^{\mathrm{a}, \mathrm{b} *}$, Giovanni Fronza ${ }^{\mathrm{b}}$, Claudio Fuganti ${ }^{\mathrm{a}, \mathrm{b}}$ and Fabio Parmeggiani ${ }^{\mathrm{a}}$ \\ ${ }^{a}$ Dipartimento di Chimica, Politecnico di Milano, Materiali, Ingegneria Chimica, Milano, Italy; ${ }^{b}$ Istituto \\ di Chimica del Riconoscimento Molecolare-CNR, Milano, Italy
}

(Received 3 September 2014; accepted 18 December 2014)

Dedicated to Professor Dr Hanns-Ludwig Schmidt on the occasion of his 85th birthday.

\section{Introduction}

The stereoselective reduction of substituted carbon-carbon double bonds is a well-established and efficient method for the creation of stereogenic centres in organic chemistry. It finds extensive applications in the synthesis of enantiomerically pure intermediates for active pharmaceutical ingredients [1]. The biocatalysed version of this reaction requires the presence of a suitable electron-withdrawing group (EWG) on the double bond. The most effective are the carbonyl groups of aldehydes and ketones, the nitro and imido functionalities [2].

Until 10 years ago, this bioreduction was usually carried out by using the resting cells of baker's yeast (BY, Saccharomyces cerevisiae) [3,4], which could be easily grown and used with-out the need of cofactors. In the last decade many enzymes responsible for the $\mathrm{C}=\mathrm{C}$ reductions have been overexpressed and made available for application in organic synthesis $[3,4]$. Most of these enzymes, including those active in BY, are called ene reductases (EC 1.6.99.1) [5]; those belonging to the superfamily of old yellow enzymes (OYEs) are the best known and most carefully investigated [6]. OYEs are characterised by the presence of a flavin mononucleotide 

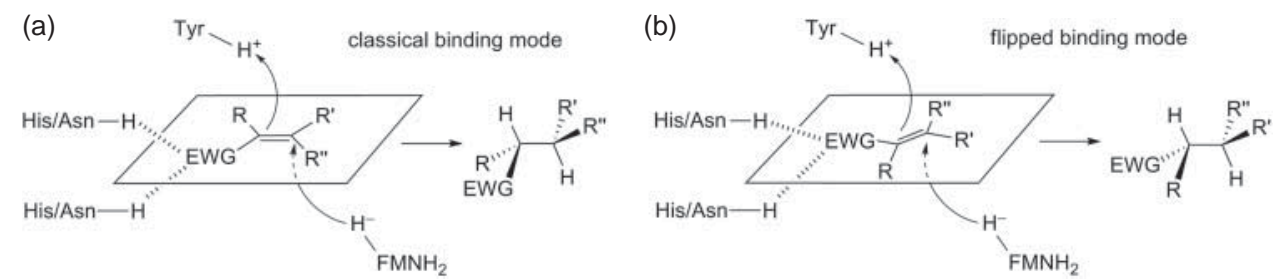

Figure 1. Mechanism of OYE-mediated reduction of $\mathrm{C}=\mathrm{C}$ double bonds, according to two possible substrate-binding modes.

(a) CHLOROESTERS<smiles>[R]C=C(Cl)C(=O)OC</smiles>

$(E)$ - and $(Z)-1$<smiles>[R]C(=CC(=O)OC)C(=O)OC</smiles>

$(E)$ - and (Z)-2 (b) DIESTERS<smiles>[R]OC(=O)C=C(C)C(=O)OCC</smiles>

$(E)$ - and $(Z)-3$ (c) CYANOESTERS<smiles>[R]OC(=O)C=C([R])C=[N+]=CC([R])=CC(=O)O[R]</smiles>

$(E)$ - and $(Z)-4$
$(E)-5$

Figure 2. Difunctionalised alkenes submitted to biocatalysed reductions.

$\left(\mathrm{FMNH}_{2}\right)$ prosthetic group, which imparts a yellow colour to the purified protein samples when it is in its oxidised form (FMN). It has been established that the substrate binds to the enzyme active site, thanks to the fact that the EWG, which is necessary for activating the alkene towards reduc-tion, is able to establish hydrogen bonds with certain amino acid residues of the enzymebinding pocket (Figure 1).

A hydride is delivered by the reduced $\mathrm{FMNH}_{2}$ prosthetic group to the carbon atom in position $\beta$ with respect to the EWG, and a proton is delivered by the tyrosine residue of the active site to the $\alpha$ carbon atom on the opposite stereoheterotopic face of the olefin, thus resulting in a stereospecific anti hydrogen addition. The final stereochemical outcome also depends on which stereoheterotopic faces of the alkene are available for hydride and proton addition, respectively, according to the orientation of the substrate in the binding pocket. Up to now, two possibilities have been described: a 'classical' and a 'flipped' binding modes [7] (Figure 1(a) and 1(b)).

The mechanism and the stereochemical course of the OYE-mediated reactions have been extensively investigated, in order to define the following aspects: (i) exception to anti hydro-gen addition; (ii) identification of the EWG involved in the hydrogen bonds within the active site, when more than one EWG is linked to the olefinic carbon atoms and (iii) effect of the stereochemistry of the starting alkenes and of the nature of substituents on the enantioselectivity of the reaction.

It is well established that deuterium labelling is a powerful tool for mechanism elucidation, and we have used it to address some open issues related to OYE-mediated reactions of the difunctionalised alkenes reported in Figure 2. The bioreduction of chloroesters $(E)$ - and $(Z)$-1, diesters $(E)$ - and $(Z)-\mathbf{2}$ and $\mathbf{3}$, cyanoesters $(E)$ - and $(Z)-\mathbf{4}$ and $(E)-\mathbf{5}$ afforded interesting results in terms of enantioselectivity that required a careful investigation. The conclusions of these studies using deuterium labelling are herein collected, in order to show the increase of knowledge brought about by this technique on the mechanism and stereochemistry of bioreductions.

\section{Results and discussion}

\subsection{Stereospecificity of hydrogen addition}

In most cases, OYE-mediated reductions generally occur according to an anti hydrogen addition. We used deuterium labelling to confirm this mechanism and explain the enantioselectivity observed in some of our biotransformations. 


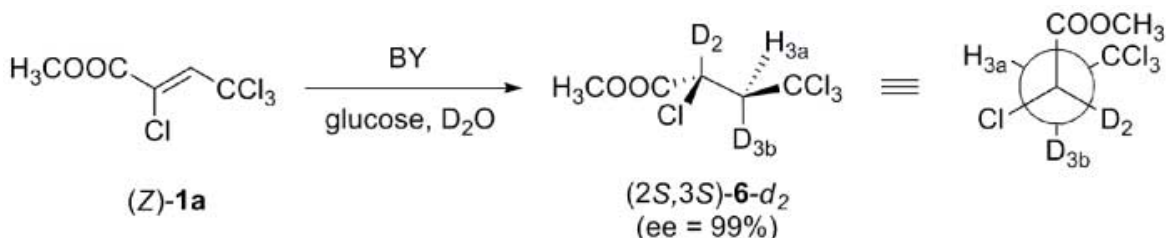

Figure 3. BY-mediated reduction of (Z)-1a in the presence of ${ }^{2} \mathrm{H}_{2} \mathrm{O}$.

Chloroesters of type 1 were submitted to BY and OYE1-3 mediated reductions [8-12]: all the $(Z)$-substrates gave the $(S)$-enantiomer of the corresponding reduced products in high yield and enantiomeric excess (ee) values, whereas the $(E)$-derivatives were converted very poorly into the $(R)$-compounds showing low enantiomeric enrichment.

The stereochemistry of hydrogen addition in the BY-mediated reduction of these chloroesters was investigated by performing the reaction of compound $(Z)-\mathbf{1 a}\left(R=\mathrm{CCl}_{3}\right)$ in the presence of deuterated water $(5 \%)$. Under these reaction conditions two deuterium atoms were added to the $\mathrm{C}=\mathrm{C}$ bond, affording the reduced product $\mathbf{6}$ (Figure 3 ).

The ${ }^{2} \mathrm{H}$ NMR spectrum of $6{ }^{2} \mathrm{H}_{2}$ showed that the two deuterium atoms had been incorporated in positions 2 and 3 with high stereoselectivity. As a matter of fact, only one of the $\mathrm{C}_{3}$ methylene protons $\left(\delta \mathrm{H}_{3 \mathrm{a}}=3.58 \mathrm{ppm}, \delta \mathrm{H}_{3 \mathrm{~b}}=2.92 \mathrm{ppm}\right.$ in benzene solution), that is, $\mathrm{H}_{3 \mathrm{~b}}$ appeared to be enriched in the deuterium nucleus. The assignment of the NMR signals of $\mathrm{H}_{3 \mathrm{a}}$ Vs. $\mathrm{H}_{3 \mathrm{~b}}$ was necessary in order to determine the absolute configuration of $\mathrm{C}_{3}$. The vicinal coupling constants $J\left(\mathrm{H}_{3 \mathrm{a}}, \mathrm{H}_{2}\right)$ and $J\left(\mathrm{H}_{3 \mathrm{~b}}, \mathrm{H}_{2}\right)$ resulted to be 7.9 and $3.8 \mathrm{~Hz}$, respectively, in the ${ }^{1} \mathrm{H}$ NMR spectrum, showing that the molecule had a preferential conformation, with the nuclei $\mathrm{H}_{3 \mathrm{a}}$ and $\mathrm{H}_{2}$ in a pseudo-anti oriented arrangement. Energy $a b$ initio calculations showed that this conformation was an absolute minimum for the molecule. Thus, $S$ configuration could be assigned to $\mathrm{C}_{3}$, and anti addition could be confirmed. Thus, the opposite enantioselectivity observed for $(E)$ - and $(Z)$-substrates was attributed to a different binding mode of the starting alkene in the enzyme active site: flipped for the $(Z)$-compounds and classical for the $(E)$ isomers.

In a further development of our research, we investigated the OYE-mediated reduction of 2substituted methyl fumarates and maleates $(E)$ - and $(Z)-2$ with alkyl chains of increasing length at $\mathrm{C}_{2}$, alkyl mesaconates and citraconates $(E)$ - and $(Z)-3$ showing ester moieties longer than $\mathrm{CH}_{3}$ [13], and cyanoesters $(E)$ - and $(Z)-4$ characterised by substituents of increasing steric hindrance at $C_{2}$ [14], in order to evaluate the effects due to the stereochemistry of the starting alkene and the nature of substituents. High values of enantioselectivity and conver-sions were obtained with compounds $(E)-\mathbf{2}$, and $(Z)-\mathbf{3}$, affording, respectively, the $(S)$-, and (R)-enantiomers of the corresponding reduced products. Derivatives $(Z)-\mathbf{2}$ and $(E)-\mathbf{3}$ were not converted by the enzymes. The reduction of cyanoesters 4 with increasing steric hindrance at $\mathrm{C}_{\alpha}$ and a $\mathrm{COOCH}_{3}$ moiety was favoured with $(E)$-starting alkenes, and afforded the cor-responding $(S)$ cyanoalkanoates with high ee values. The increase of the steric hindrance of the alkyl residue of the ester moiety had a different effect: the $(Z)$-stereoisomer became the best substrate and the $(R)$-enantiomer was obtained starting from either $(E)$ - or $(Z)$-starting material.

The interpretation of these data required the definition of the mechanism of hydrogen addition. With this aim, the OYE1-mediated reduction of $(E)$ - and $(Z)-\mathbf{2 a},(E)-\mathbf{2 b},(Z)-\mathbf{3 a},(E)$ and $(Z)-\mathbf{4 a},(Z)-\mathbf{4 b - d}$ (Figure 4) was carefully considered.

When isolated OYEs are used, the incorporation of two deuterium atoms is possible in ${ }^{2} \mathrm{H}_{2} \mathrm{O}$ solution by using $(R)$-NADPD, regenerated in situ by Thermoanaerobium brockii alcohol dehydrogenase (TBADH) and $i-\mathrm{PrOH}_{-}{ }^{2} \mathrm{H}_{8}[15]$. 
<smiles>[R]C(=CC(=O)OC)C(=O)OC</smiles>

$(E)$ - and (Z)-2

a $\mathrm{R}=\mathrm{CH}_{3}$

b $\mathrm{R}=\mathrm{CH}_{2} \mathrm{CH}_{3}$<smiles>[R]OC(=O)C=C(C)C(=O)O[R]</smiles>

$(E)$ - and $(Z)-3$

a $\mathrm{R}^{\prime}=\mathrm{CH}_{2} \mathrm{CH}_{3}$<smiles>[R]OC(=O)C([R])=CC#N</smiles>

$(E)$ - and $(Z)-4$

a $\mathrm{R}=\mathrm{CH}_{3}, \mathrm{R}^{\prime}=\mathrm{CH}_{3}$

b $\mathrm{R}=\mathrm{CH}_{2} \mathrm{CH}_{3}, \mathrm{R}^{\prime}=\mathrm{CH}_{3}$

c R $=\left(\mathrm{CH}_{2}\right)_{3} \mathrm{CH}_{3}, \mathrm{R}^{\prime}=\mathrm{CH}_{3}$

d $\mathrm{R}=\mathrm{CH}_{3}, \mathrm{R}^{\prime}=\left(\mathrm{CH}_{2}\right)_{3} \mathrm{CH}_{3}$

Figure 4. Substrates submitted to deuterium labelling to investigate the stereospecificity of hydrogen addition.

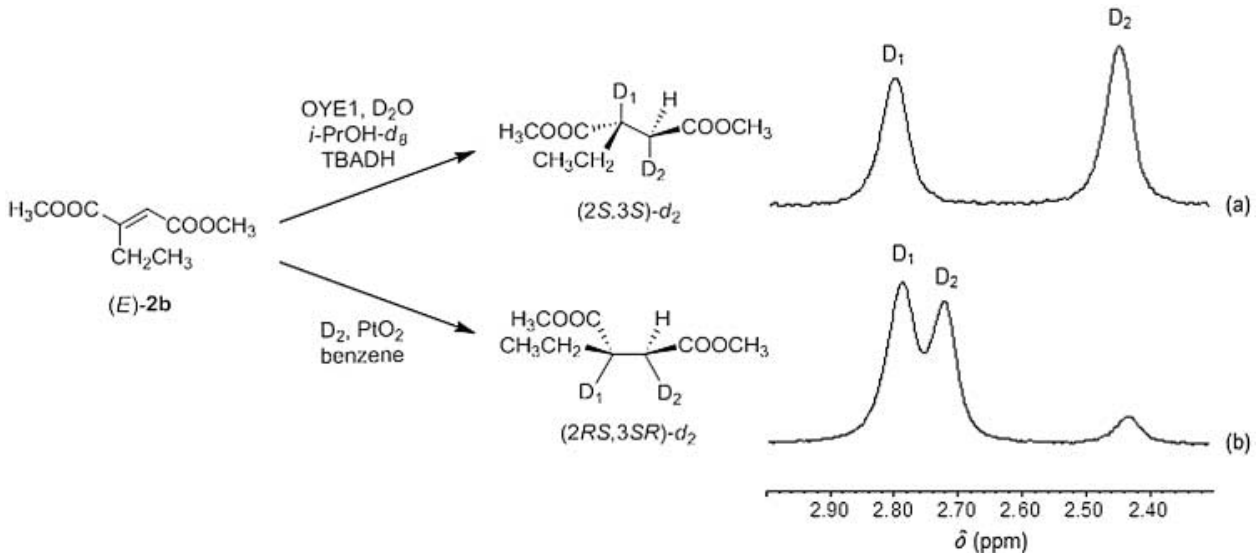

Figure 5. $\quad{ }^{2} \mathrm{H}$ NMR spectra of the dideuterated compound obtained by OYE1-mediated reduction of $(E)-2 \mathbf{b}(a)$ and the dideuterated compound obtained by chemical deuteration of $(E)-\mathbf{2 b}(\mathrm{b})$.

Unlike chloroesters, these diesters and cyanoesters could be submitted to chemical deuteration to prepare reference compounds. The use of deuterium gas in the presence of $\mathrm{PtO}_{2}$ or $\mathrm{Pt} / \mathrm{C}$ in benzene solution afforded the dideuterated derivatives by a syn addition mechanism. The ${ }^{2} \mathrm{H}$ NMR spectra showed that the dideuterated products obtained by biocatalysed reduction of the $(E)$-alkenes did not match those obtained by chemical syn deuteration of the same starting substrates, thus confirming anti hydrogen addition. The results obtained for diester $(E)-\mathbf{2 a}$ are shown in Figure 5.

The ${ }^{2} \mathrm{H}$ NMR spectrum reported in Figure 5(b) shows traces of the $(2 R S, 3 R S)$ diastereoisomer due to the occurrence of a partial scrambling during the process of metal-catalysed deuteration. This phenomenon was found to be of a higher extent when deuteration was performed by using $\mathrm{Pd} / \mathrm{C}$.

The opposite enantioselectivity of the bioreduction of $(E)$ - and $(Z)$-stereoisomers could be attributed to a different binding mode (either classical or flipped) of the substrate to the enzyme active site.

\subsection{Exceptions to the anti mechanism: the case of perillaldehyde}

The reduction of perillaldehyde 7 mediated by BY became of interest for the production of the relevant natural flavour dihydroperillaldehyde. The results of the bioreduction induced us to carefully investigate the mechanism of the regioselective reduction of the conjugate double bond [16]. 


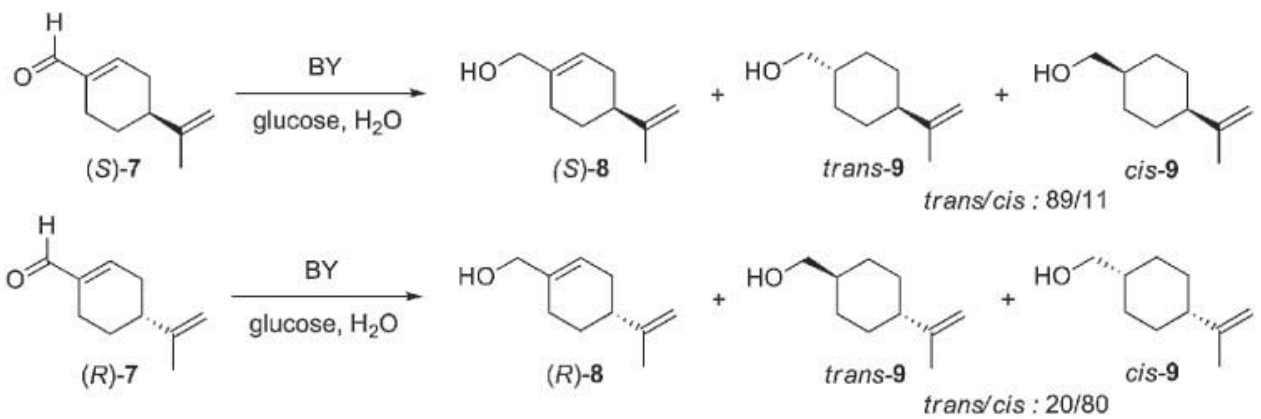

Figure 6. BY-mediated reduction of $(S)$ - and $(R)$-perillaldehyde.

The incubation of fermenting BY with $(S)-7$ afforded a mixture of perillyl alcohol $\mathbf{8}(51 \%)$ and saturated compounds trans-9 and cis-9 (48 \%) in 89:11 ratio, respectively, (Figure 6). The concomitant reduction of the carbonyl group to the primary alcohol is due to the presence of endogenous alcohol dehydrogenases in fermenting BY. When the procedure was applied on $(R)-7$, the ratio of allylic alcohol/saturated alcohols was the same, but the trans/cis ratio of the products obtained by double bond reduction was reversed: cis-9 was the major diastereoisomer with a cis/trans ratio of 80:20.

The origin of the hydrogen atoms added to the double bond was studied by deuterium labelling experiments by performing the BY-catalysed reaction in deuterated water: the analysis of the ${ }^{2} \mathrm{H}$ NMR spectra clearly demonstrated a different mechanism of the biohydrogenation of the two enantiomers of perillaldehyde.

Compound trans $-9-{ }^{2} \mathrm{H}_{3}$, obtained from $(S)$-perilladehyde, showed only one deuterium atom in position 2 , in a trans-diaxial arrangement with respect to the deuterium linked to $C_{1}$, as it was deduced from the large coupling constant of $12.5 \mathrm{~Hz}$ occurring between the corresponding hydrogen nuclei in the proton spectrum. This is in agreement with an anti deuterium addition (Figure 7) being the substrate bound to the active site according to a flipped-binding mode.

Compound cis-9- ${ }^{2} \mathrm{H}_{3}$ obtained from $(R)-7$ showed two deuterium atoms in position 2 , one equatorial and the other axial, thus suggesting the occurrence of two different hydrogena-tion mechanisms on this particular substrate. As a matter of fact, Figure 7 shows that the cis stereoisomer can be obtained from $(R)-7$ either by anti addition to the $\mathrm{C}=\mathrm{C}$ of the sub-strate in a flipped-binding mode, or by syn addition to the substrate in a classical-binding mode.

The two enantiomers of perilladehyde were characterised by an anomalous behaviour also when the bioreduction was performed by using the rat NADP-dependent leukotriene B4 12hydroxydehydrogenase (Ltb4dh), an isolated enzyme which is capable of reducing activated alkenes at the expense of NADPH in the absence of a flavin cofactor [15]. Ltb4dh reduced both enantiomers of perillaldehyde to the same cis-product. To explain this unexpected result, the stereochemical courses of perillaldehyde reductions by Ltb4dh were investigated by deu-terium labelling followed by ${ }^{2} \mathrm{H}$ NMR analysis. Both $(R)$ - and $(S)$-perillaldehyde were reduced by GSTLtb4dh in ${ }^{2} \mathrm{H}_{2} \mathrm{O}$ solvent in the presence of $(R)$-NADPD, regenerated in situ with the TBADH/i$\mathrm{PrOH}_{-}{ }^{2} \mathrm{H}_{8}$ system described above. Anti-addition of ${ }^{2} \mathrm{H}_{2}$ to the conjugated alkene of $(R)$ perillaldehyde in a flipped-binding mode afforded $c i s-9-d_{3}$ with the introduc-tion of two equatorial deuterium atoms at $\mathrm{C}_{1}$ and $\mathrm{C}_{2}$, as it was confirmed by ${ }^{2} \mathrm{H}$ NMR data. Syn addition of ${ }^{2} \mathrm{H}_{2}$ to the conjugated $\mathrm{C}=\mathrm{C}$ bond of $(S)$-perillaldehyde in a flipped-binding mode gave cis-9- ${ }^{2} \mathrm{H}_{3}$ with the introduction of an axial deuterium at $\mathrm{C}_{2}$ according to the analysis of ${ }^{2} \mathrm{H}$ NMR data, thus showing that the stereospecificity of hydrogen addition was different. 


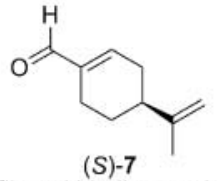

flipped binding mode

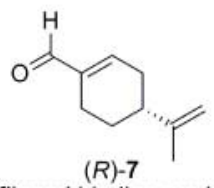

flipped binding mode

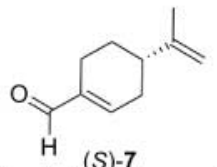

classical binding mode

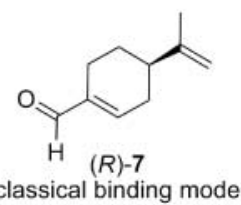

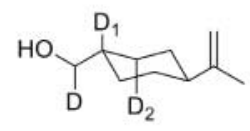

trans-9- $d_{3}$
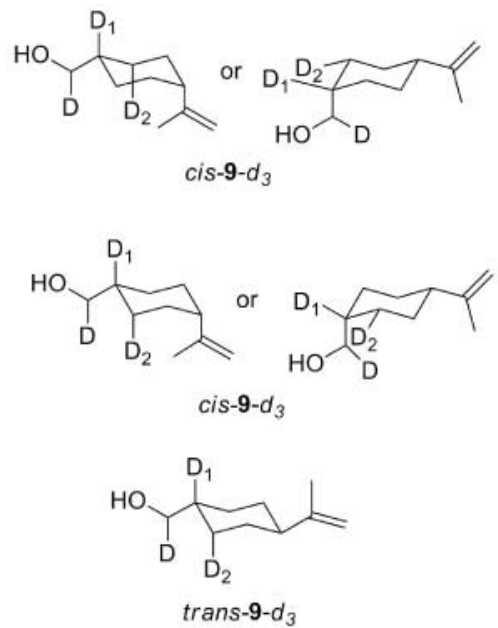
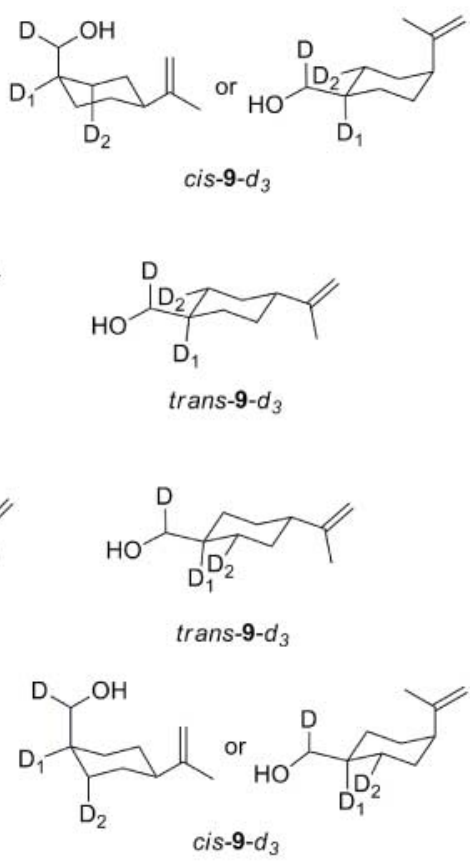

Figure 7. Deuterated compounds obtained by BY-reduction on $(S)$ and $(R)$-perillaldehyde.

\subsection{Identification of the EWG involved in the substrate binding within the enzyme active site}

$\alpha, \beta$-Unsaturated aldehydes and ketones together with nitroalkenes and maleimides are typi-cal substrates for ERs, whereas, for example, the bioreduction of $\alpha, \beta$-unsaturated carboxylic acids and derivatives has been rarely obtained [17], because the electron-withdrawing effect of a single carboxylic moiety is generally not enough to promote ER-mediated reduc-tion.

The structural characteristics of the EWGs which are needed for the double bond reduction are still to be clearly defined, in order to establish the synthetic potential of this procedure and to include it into the enzyme tool box available to synthetic chemists. With this aim, we have recently investigated the OYE-mediated reduction of difunctionalised substrates reported in Figure 2, in order to establish whether the presence of a second EWG besides the carboxylic moiety could promote the $\mathrm{C}=\mathrm{C}$ bioreduction $[13,14,18-20]$. The comprehension of the different stereochemical outcome of the $(E)$ and $(Z)$ stereoisomers of the same compound required the identification of the EWG involved in the formation of the hydrogen bonds in the enzyme active site.

The use of stoichiometric NADH and ${ }^{2} \mathrm{H}_{2} \mathrm{O}$ as a solvent of the OYE-mediated reactions promotes the incorporation of a hydrogen atom at the carbon atom in the $\beta$ position (from NADH) and of a deuterium atom at the carbon atom in the $\alpha$ position (from the Tyr residue) with respect to the activating group, that is, the one which is involved in the formation of hydrogen bonds with the amino acid residues within the active site of the enzyme. The analysis of the ${ }^{1} \mathrm{H}$ NMR spectrum of the monodeuterated reduced compound allows the position of the deuterium atom to be established. In Figure 8 the proton NMR spectrum of the monodeuterated compound obtained 


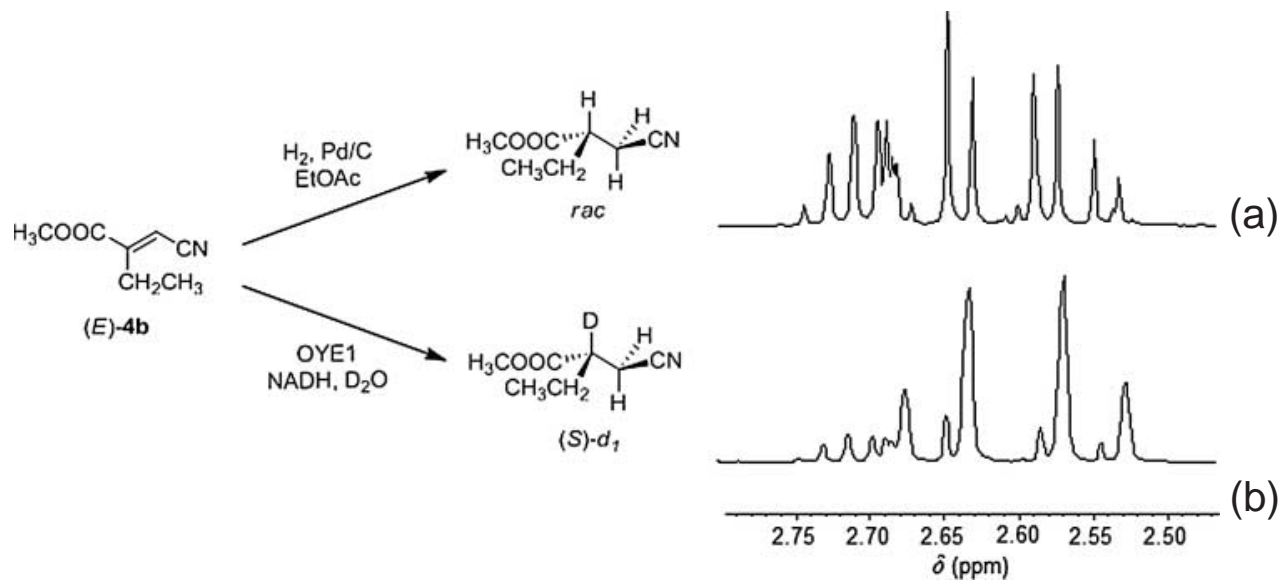

Figure 8. $\quad{ }^{1} \mathrm{H}$ NMR spectra of the compound obtained by chemical hydrogenation of $(E)-\mathbf{4 b}(\mathrm{a})$, and the monodeuterated compound obtained by OYE-mediated reduction of $(E)-\mathbf{4 b}(\mathrm{b})$.

from $(E)-\mathbf{4 b}$ by OYE-mediated reduction is compared with that of the corresponding racemic derivative obtained by chemical hydrogenation. The disappearance of the multiplet due to the hydrogen atom linked to $\mathrm{C}_{2}$ demonstrates that the ester moiety is the EWG involved in the hydrogen bonds within the enzyme active site.

The experimental data showed that trisubstituted $\alpha, \beta$-difunctionalised alkenes, such as diesters $(E)$ - and (Z)-2 and 3, cyanoesters $(E)$ - and $(Z)-\mathbf{4}$ and $(E)$-5, have a preference for binding to the enzyme active site through the EWG linked to the prostereogenic olefinic carbon atom. The most reactive-binding mode does not seem to be influenced by the electronic and geometric characteristics of the activating functional group. Even the linear nitrile group can afford a suit-able hydrogen bond interaction within the enzyme active site. Only a few exceptions have been identified [14,21].

\subsection{Definition of the most reactive substrate-binding mode}

We collected the information on the hydrogen addition mechanism, on the EWG involved in the substrate-binding mode, and on the absolute configuration of the reduced products for sev-eral activated alkenes, and we made some considerations on the orientation of the alkene in the binding pocket.

The stereochemistry of the starting alkene and the steric hindrance of the EWG and of the substituents linked to the olefinic carbon atoms were related to the most reactive-binding mode of the substrate, which establishes the enantioselectivity of the process. The conclusions are summarised in Figure 9 for unsaturated trisubstituted alkenes, whose activating EWG (either a carbonyl, or a carboxyl, or a nitrile moiety) is linked to the prostereogenic carbon atom (one of the substituent at $\mathrm{C}_{\beta}$ is a hydrogen atom). The preferred reactive-binding mode seems to be determined by the relative size of the EWG and of the substituent linked to $\mathrm{C}_{\alpha}(\alpha)$ : if this substituent is bulky or more hindered ( $\mathrm{L}$, large), then a flipped-binding mode is preferred; if it is less bulky (S, small), then a classical-binding mode is adopted. Having the substrate adopted a flipped binding, the alkene stereoisomer best reduced is usually the one with the large substituent at $\mathrm{C}_{\beta}(\mathrm{L})$ on the same side of the EWG. If the classical-binding mode is preferred, the alkene stereoisomer best reduced is the one with the large substituent at $\mathrm{C}_{\beta}(\mathrm{L})$ on the opposite side of the EWG. 


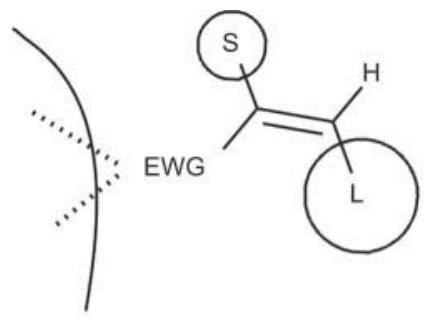

classical binding mode

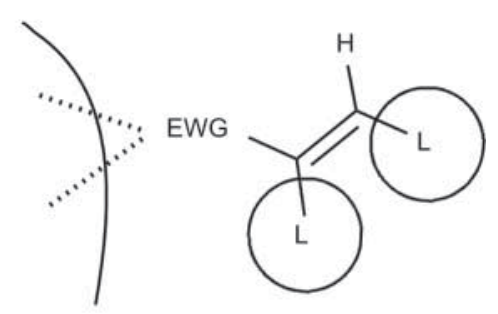

flipped binding mode

Figure 9. Model for the OYE-mediated reductions of trisubstitued alkenes activated by an EWG linked to the prosterogenic carbon atom.

\section{Conclusions}

Deuterium labelling is a powerful technique to obtain information on the mechanism and stereochemistry of OYE-mediated reductions. The definition of the mechanism of hydrogen addition and the identification of the effective EWG are essential details to explain the enantioselectivity of the reaction in relation to the stereochemistry of the starting alkene, and to the nature of the substituents. The information gathered by means of labelling experiments allowed an empirical model to be obtained for the identification of new optimal substrates for biocatalysed reductions. The aim is to obtain a control on enantioselectivity and conversion through a wise structural definition of the starting compound.

\section{Disclosure statement}

No potential conflict of interest was reported by the authors.

\section{References}

[1] Johnson NB, Lennon IC, Moran PH, Ramsden JA. Industrial-scale synthesis and applications of asymmetric hydrogenation catalysts. Acc Chem Res. 2006;40:1291-1299.

[2] Gatti FG, Parmeggiani F, Sacchetti A. Synthetic strategies based on C=C bioreductions for the preparation of biologically active molecules (Chapter 3). In: Brenna E, editor. Synthetic methods for biologically active molecules - exploiting the potential of bioreductions. Weinheim: Wiley-VCH; 2014. p. 49-84.

[3] Csuk R, Glänzer BI. Baker's yeast mediated transformations in organic chemistry. Chem Rev. 1991;91:49-97.

[4] Servi S. Baker's yeast as a reagent in organic synthesis. Synthesis. 1990;1:1-25.

[5] Toogood HS, Scrutton NS. New developments in 'ene'-reductase catalysed biological hydrogenations. Curr Opin Chem Biol. 2014;19:107-115.

[6] Toogood HS, Gardiner JM, Scrutton NS. Biocatalytic reductions and chemical versatility of the Old Yellow Enzyme family of flavoprotein oxidoreductases. ChemCatChem. 2010;2:892-914.

[7] Brenna E, Gatti FG, Monti D, Parmeggiani F, Serra S. Stereochemical outcome of the biocatalysed reduction of activated tetrasubstituted olefins by Old Yellow Enzymes 1-3. Adv Synth Catal. 2012;354:105-112.

[8] Brenna E, Fronza G, Fuganti C, Monti D, Parmeggiani F. Enantioselective C $=\mathrm{C}$ bond reduction of unsaturated $\alpha$-chloro esters by old yellow enzymes. J Mol Catal B: Enzym. 2011;73:17-21.

[9] Utaka M, Konishi S, Ohkubo T, Tsuboi S, Takeda A. A facile synthesis of optically pure L-armentomycin and its D-isomer. Highly enantioselective reduction of the $\mathrm{C}=\mathrm{C}$ double bond of methyl (E)- and (Z)-2,4,4-trichloro-2butenoate by using baker's yeast. Tetrahedron Lett. 1987;28:1447-1449.

[10] Utaka M, Konishi S, Mizouka A, Ohkubo T, Sakai T, Tsuboi S, Takeda A. Asymmetric reduction of the prochiral carbon-carbon double bond of methyl 2-chloro-2-alkenoates by use of fermenting bakers' yeast. J Org Chem. 1989;54:4989-4992.

[11] Brenna E, Gatti FG, Manfredi A, Monti D, Parmeggiani F. Biocatalyzed enantioselective reduction of activated $\mathrm{C}=\mathrm{C}$ bonds: synthesis of enantiomerically enriched $\alpha$-halo- $\beta$-arylpropionic acids. Eur $\mathrm{J}$ Org $\mathrm{Chem}$. 2011;2011:4015-4022.

[12] Brenna E, Gatti FG, Manfredi A, Monti D, Parmeggiani F. Enoate reductase-mediated preparation of methyl (S)-2bromobutanoate, a useful key intermediate for the synthesis of chiral active pharmaceutical ingredients. Org Process Res Dev. 2012;16:262-268. 
[13] Brenna E, Gatti FG, Manfredi A, Monti D, Parmeggiani F. Steric effects on the stereochemistry of Old Yellow Enzyme-mediated reductions of unsaturated diesters: flipping of the substrate within the enzyme active site induced by structural modifications. Adv Synth Catal. 2012;354:2859-2864.

[14] Brenna E, Gatti FG, Manfredi A, Monti D, Parmeggiani F. Old Yellow Enzyme-mediated reduction of $\beta$-cyano$\alpha, \beta$-unsaturated esters for the synthesis of chiral building blocks: stereochemical analysis of the reaction. Catal Sci Technol. 2013;3:1136-1146.

[15] Bougioukou DJ, Stewart JD. Opposite stereochemical courses for enzyme-mediated alkene reductions of an enantiomeric substrate pair. J Am Chem Soc. 2008;130:7655-7658.

[16] Fronza G, Fuganti C, Pinciroli M, Serra S. Stereochemical aspects of the bioreduction of the conjugated double bond of perillaldehyde. Tetrahedron: Asymmetry. 2004;15:3073-3077.

[17] Stueckler C, Winkler CK, Bonnekessel M, Faber K. Asymmetric synthesis of (R)-3-hydroxy-2-methylpropanoate ('Roche Ester') and derivatives via biocatalytic C=C bond reduction. Adv Synth Catal. 2010;352:2663-2666.

[18] Walton AZ, Conerly WC, Pompeu Y, Sullivan B, Stewart JD. Biocatalytic reductions of Baylis-Hillman adducts. ACS Catal. 2011;1:989-993.

[19] Pompeu YA, Sullivan B, Walton AZ, Stewart JD. Structural and catalytic characterization of Pichia stipitis OYE 2.6, a useful biocatalyst for asymmetric alkene reductions. Adv Synth Catal. 2012;354:1949-1960.

[20] Tasnádi G, Winkler CK, Clay D, Sultana N, Fabian WMF, Hall M, Ditrich K, Faber K. A substrate-driven approach to determine reactivities of $\alpha, \beta$-unsaturated carboxylic esters towards asymmetric bioreduction. Chem Eur J. 2012;18:10362-10367.

[21] Brenna E, Crotti M, Gatti PG, Manfredi A, Monti D, Parmeggiani F, Pugliese A, Zampieri D. Rationalisation of the stereochemical outcome of ene-reductase-mediated bioreduction of $\alpha, \beta$-difunctionalised alkenes. J Mol Catal B: Enzym. 2014;101:67-72. 